\title{
Herida Penetrante Cervical En Pediatría
}

Adriana M Simons ${ }^{1}$, Ezequiel Monteverde ${ }^{2}$, Andrea Francavilla $^{3}$, Alejandro Gattari ${ }^{4}$, Deborah Turina ${ }^{5}$, Analia Costas $^{6}$, Pilar A Lopez ${ }^{7}$, Pablo Neira ${ }^{8}$

\section{RESUMEN}

El traumatismo penetrante de cuello (TPC) es poco frecuente en la edad pediátrica. Las lesiones vasculares por TPC contribuyen significativamente a la morbimortalidad, siendo la hemorragia severa la causa más habitual de muerte temprana. La escasa información sobre el tema y la complejidad diagnóstica y terapéutica que estos pacientes pueden presentar motivación a considerar los siguientes casos asistidos en una unidad de cuidados intensivos pediátricos (UCIP) en un periodo de 5 años. Los casos relatados presentaron evoluciones distintas, debido a diferentes mecanismos de producción. Dos de los mismos requirieron exploración quirúrgica de urgencia con intervenciones según los hallazgos intraoperatorios. En el tercer caso, debido al buen estado general y estabilidad hemodinámica del paciente, se decidió mantener conducta quirúrgica expectante con resultados satisfactorios. La mortalidad de estos pacientes es elevada durante la hora de oro, ya que los resultados terapéuticos dependen de un alto grado de sospecha de las lesiones.

Palabras claves: Herida penetrante de cuello, Herida de arma de fuego, Lesión de vía aérea, Lesiones vasculares región cervical, Trauma pediátrico.

\section{Abstract}

Penetrating neck trauma (PNT) is infrequent in children. Penetrating neck trauma-related vascular injuries contribute significantly to morbidity and mortality, with severe bleeding being the most common cause of early death. Scarcity of information on the subject, and the diagnostic and therapeutic complexity that these patients present, motivate the consideration for presentation of the following cases admitted to a pediatric intensive care unit (PICU) in a 5-year period. These cases showed different evolution and mechanism of injury. Two of them required emergency surgical exploration with final procedures derived from intraoperative findings. In the third case, given his good general condition and hemodynamic stability, it was decided to maintain a non-operative approach with satisfactory results. The mortality of these patients is high during the golden hour, since the therapeutic results depend on a high-degree suspicion index.

Keywords: Airway injury, Firearm injury, Pediatric trauma, Penetrating neck injury, Vascular injuries cervical region.

Panamerican Journal of Trauma, Critical Care \& Emergency Surgery (2021): 10.5005/jp-journals-10030-1312

\section{INTRODUCCIÓN}

Se define al traumatismo penetrante de cuello (TPC) como toda herida que penetra más allá del músculo cutáneo cervical. Es una entidad clínica habitualmente de urgencia y que suele asociarse a lesiones en territorios anexos. ${ }^{1}$ Es una entidad muy poco frecuente en la edad pediátrica, con una prevalencia que oscila entre $0.02 \%{ }^{2}$ y $0.28 \%{ }^{3}$ según las series de mayor tamaño, por lo que la información sobre epidemiología, patrón lesional y manejo es muy escasa. ${ }^{4} \mathrm{La}$ edad mediana oscila entre 7 y 8 años, sin distribución diferencial por género y más del $95 \%$ se deben a lesiones por arma de fuego u objetos punzocortantes. ${ }^{5}$

Las lesiones vasculares por TPC contribuyen significativamente a la morbimortalidad, siendo la hemorragia severa la causa más frecuente de muerte temprana. ${ }^{6}$ El daño a la faringe o al esófago ocurre en $5-15 \%$ de los casos, la laringe o la tráquea se lesiona en 4-12\% y las lesiones de nervios principales en 3-8\%. ${ }^{7}$ En una base de datos argentina de trauma, ${ }^{8}$ sobre 10,310 casos pediátricos, se hallaron lesiones cervicales en el 1.2\%, en su mayoría superficiales. El $62 \%$ tuvo al menos una lesión en otra región, lo cual se asoció a mayor severidad (ISS $\geq 16$ ): $10.7 \%$ vs $3.9 \%$ y mortalidad $(4.9 \%$ vs $1.3 \%)$.

Dada la baja prevalencia en este rango etario, el manejo asistencial se extrapola de la experiencia en adultos. ${ }^{6,9}$ El objetivo de esta comunicación es presentar la evolución clínica de tres pacientes pediátricos con TPC.
${ }^{1}$ Unidad de Cuidados intensivos Pediatricos del Hospital Interzonal Espacializado Materno infantil Dr. Tetamanti, Mar del Plata, Buenos Aires, Argentina

${ }^{2-8}$ Unidad de Cuidados Intensivos pediatricos del Hospital de Niños Ricardo Gutierres, CABA, Buenos Aires, Argentina

Corresponding Author: Adriana M Simons, Unidad de Cuidados intensivos Pediatricos del Hospital Interzonal Espacializado Materno infantil Dr. Tetamanti, Mar del Plata, Buenos Aires, Argentina, Phone: +54 111531120888, e-mail: simonsadrianam@gmail.com

How to cite this article: Simons AM, Monteverde E, Francavilla A, et al. Herida Penetrante Cervical En Pediatría. Panam J Trauma Crit Care Emerg Surg 2021;10(1):46-50.

Source of support: Nil

Conflict of interest: None

\section{Presentación de Casos}

\section{Caso 1}

Paciente de 9 años, sexo femenino, previamente sana, que ingresa llevada por su familia a las 5:15 al servicio de Emergencias de un Hospital Intermedio de la Ciudad Autónoma de Buenos Aires (CABA) presentando HPC secundaria a lesión con barra metálica no sujeta mientras viajaba sin cinturón de seguridad en el asiento trasero de un automóvil. Se presenta en mal estado general, taquicárdica (120 latidos por minuto), con excitación psicomotriz,

(c) The Author(s). 2021 Open Access This article is distributed under the terms of the Creative Commons Attribution 4.0 International License (https:// creativecommons.org/licenses/by-nc/4.0/), which permits unrestricted use, distribution, and non-commercial reproduction in any medium, provided you give appropriate credit to the original author(s) and the source, provide a link to the Creative Commons license, and indicate if changes were made. The Creative Commons Public Domain Dedication waiver (http://creativecommons.org/publicdomain/zero/1.0/) applies to the data made available in this article, unless otherwise stated. 


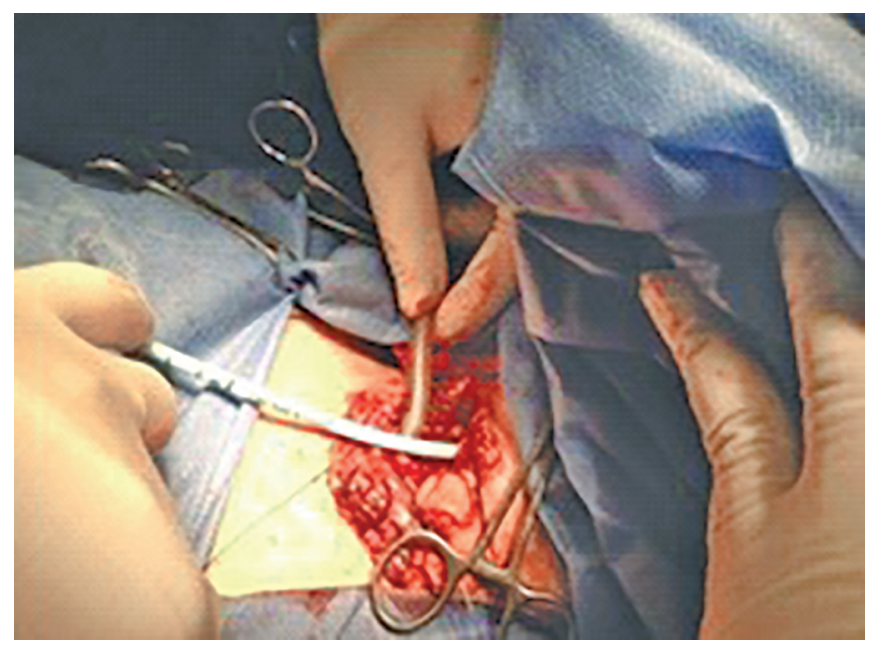

Fig. 1: Exploración quirúrgica

imposibilidad de fonar y aerorragia por cuello. Se visualiza exposición y apertura traqueal en cara anterior del cuello, por lo cual se intuba a través de dicho orificio, lográndose asegurar la vía aérea. Luego de la colocación de 2 accesos venosos periféricos se inicia reanimación con cristaloides e ingresa al quirófano con carácter de urgencia. La exploración de la herida muestra: (1) sección de la vena yugular externa derecha, que se liga; (2) sección traqueal a nivel del segundo anillo con compromiso del $75 \%$ de la circunferencia, respetando pared posterior; (3) sección de glándula tiroides a nivel del istmo y lóbulo derecho y (4) esófago sin lesiones Figure 1. Se realiza plástica traqueal, intubación orotraqueal, hemostasia de glándula tiroides y reparo de planos musculares y cutáneos logrando una hemostasia adecuada. La duración total del procedimiento fue 150 minutos.

Se traslada inmediatamente después de la cirugía al Hospital de Niños Ricardo Gutiérrez (HNRG)-centro de referencia para trauma pediátrico de la CABA-a través del servicio de emergencias del sistema público de salud. La duración del traslado fue de 30 minutos, ingresando la paciente a las 8:45 con asistencia ventilatoria mecánica (AVM), bajo sedoanalgesia y hemodinámicamente lábil. Se indica expansión con coloides y transfusión de glóbulos rojos desplasmatizados. Una vez colocado el acceso venoso central, se inicia la infusión de dopamina hasta lograr la estabilización hemodinámica, y se indica tratamiento antibiótico con clindamicina y gentamicina como profilaxis por HPC con lesión aerodigestiva. Durante la internación es evaluada por cirujanos especialistas en cabeza y cuello mediante tomografía computada, donde se observa alteración de los tejidos blandos de la región anterior del cuello, con probable afectación del istmo tiroideo y engrosamiento de los músculos infrahioideos.

La paciente requirió AVM por 25 días, con una estadía en la unidad de cuidados intensivos pediátricos (UCIP) de 27. Durante este período presentó síndrome de distress respiratorio agudo (SDRA) grave, por lo que requirió ventilación en decúbito prono por 7 días. Presentó también una neumonía asociada a la ventilación mecánica (NAV) por A. baumanii, que resolvió favorablemente con tratamiento antibiótico. La estadía total fue de 47 días y el tiempo de internación en sala general se relacionó con el destete de opioides y el control de la función tiroidea. La paciente egresó en buen estado general, respirando espontáneamente, con videodeglución y función tiroidea normales Figure 2.

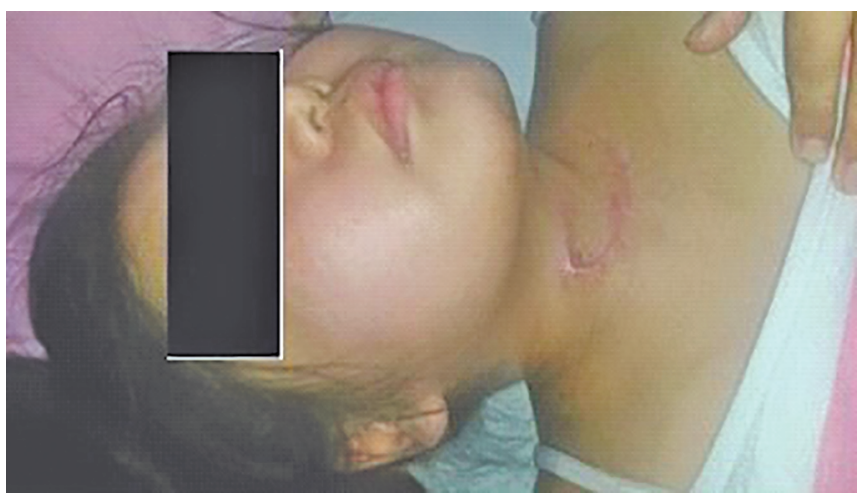

Fig. 2: Paciente el día de su egreso hospitalario

\section{Caso 2}

Paciente de sexo masculino de 14 años de edad, previamente sano, que sufre a las 14:00 una herida por arma de fuego en la región cervical lateral izquierda, luego de lo cual es trasladado por el sistema público de emergencias a un hospital general de agudos de la CABA. Ingresa en estado crítico, con shock hipovolémico por sangrado activo en el sitio de la lesión, presentando paro cardiorrespiratorio durante la atención inicial, por lo que se realiza reanimación cardiopulmonar avanzada y compresión manual del lecho sangrante, luego de lo cual es trasladado al quirófano. Presenta herida con orificio de entrada y sin orificio de salida en zonas I y II cervicales izquierdas. Durante la exploración se constata fractura conminuta de la rama horizontal izquierda de la mandíbula, lesión grave de la arteria carótida interna y lesión de vértebra cervical con daño presunto del paquete vascular vertebral. Se realiza la ligadura proximal y distal de la carótida izquierda, a pesar de lo cual el paciente continúa con hemorragia, luego se ligan la carótida externa, yugular y vertebral izquierdas, además del taponamiento del pedículo vertebral. Durante el procedimiento recibe múltiples transfusiones e infusión de vasopresores. Se traslada inmediatamente después de la cirugía al HNRG, donde ingresa a las 18:00 hemodinámicamente inestable, con AVM, recibiendo infusión continua de vasopresores, con coagulopatía -tiempo de protrombina (Quick) de 41\%-, con acidosis metabólica, hipotermia, anisocoria y midriasis paralítica izquierda. Luego de estabilizarlo, se realiza TC de cuello y cerebro con y sin contraste endovenoso, que informa: "Trayecto de un proyectil de arma de fuego ingresando en la región del maxilar inferior izquierdo con disrupción de la rama horizontal del mismo y trayecto en sentido caudal atravesando en su recorrido la región lateral del cuello con disrupción de la carótida interna. El pedículo izquierdo de la quinta vértebra cervical presenta lesión de la arteria vertebral a este nivel. Se observan fragmentos óseos en el canal neural con probable lesión de la médula espinal. Proyectil alojado en el espesor de los músculos de la región posterior del cuello a la altura de C5-C6. Hipodensidad difusa de ambos hemisferios cerebrales a predominio izquierdo, sugestivo de isquemia y edema". Figure 3 y 4 . Es evaluado por el servicio de Neurocirugía, quienes colocan catéter de fibra óptica para medición de la presión intracraneana, que muestra una presión de apertura de $71 \mathrm{~mm} \mathrm{Hg}$, con valores de presión de perfusión cerebral que oscilaban entre 0 y $10 \mathrm{~mm} \mathrm{Hg}$ (normal por edad 55-60). Posteriormente el paciente presenta midriasis paralítica bilateral. Se desestima la realización de una craniectomía descompresiva amplia por la marcada inestabilidad hemodinámica, 


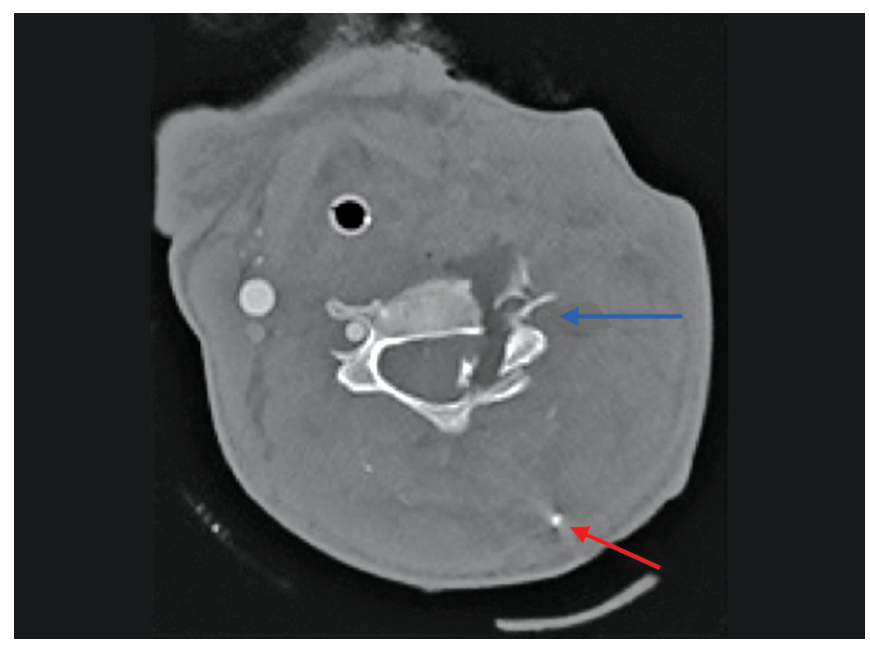

Fig. 3: Tomografía computada donde se observa lesión cervical y compromiso del canal medular. Se observa proyectil
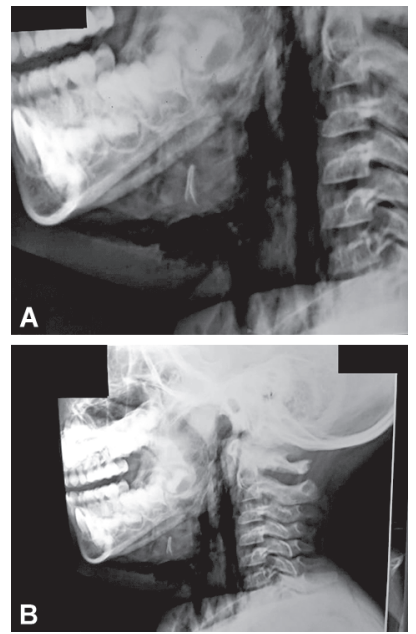

Figs $5 A$ and $B$ : Rx cervical perfil

por lo que se continúa con tratamiento médico: terapia osmolar, sostén hemodinámico, respiratorio y control de la coagulación. El paciente evoluciona con diabetes insípida, recibiendo corrección con agua libre y desmopresina. El electroencefalograma muestra patrón de bajo voltaje y el doppler transcraneano, evidencia de interrupción del flujo circulatorio cerebral. Luego de suspender la sedoanalgesia y los bloqueantes neuromusculares, se realiza evaluación por el equipo de trasplante, quienes diagnostican muerte bajo criterios neurológicos a las 24 hs del trauma. No se realizó ablación de órganos.

\section{Caso 3}

Paciente de 4 años de edad, sexo femenino, previamente sana, que es trasladada por sus familiares a un hospital general de la CABA por HPC secundaria a caída de la cama sobre objeto punzante (tornillo o clavo) en región supra-esternal. En el servicio de Emergencias la evalúan vigil y orientada, con facie dolorosa, taquicárdica (130 latidos por minuto), normotensa (tensión arterial 110/80 mm Hg) con aumento del perímetro cervical, aerorragia y enfisema subcutáneo en región cervical anterior. Se colocan dos accesos vasculares periféricos, se administra analgesia e hidratación parenteral y

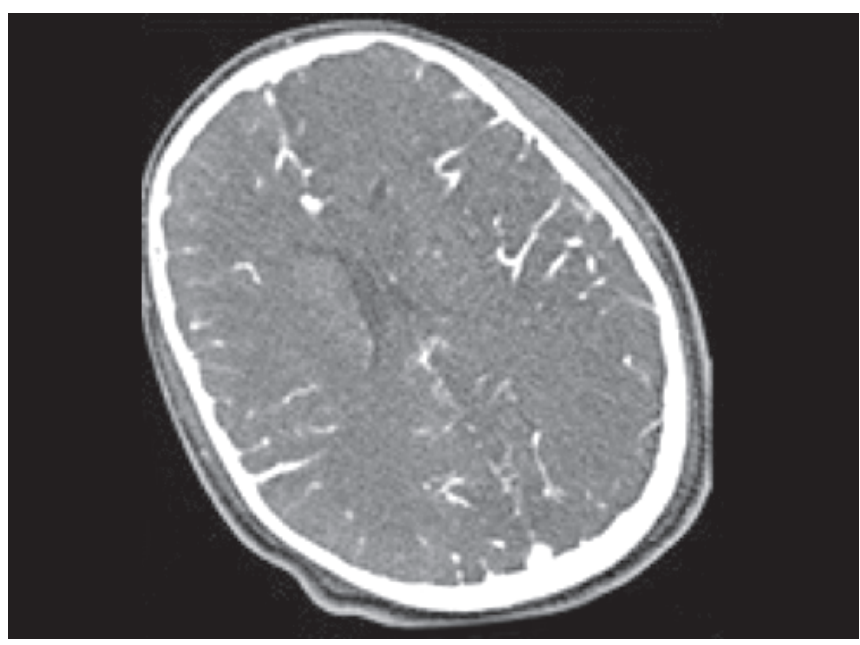

Fig. 4: Tomografía computada de cerebro con contraste endovenoso post colocación de catéter de presión intracraneana: sistema ventricular supratentorial colapsado, hipodensidad difusa de ambos hemisferios, especialmente del izquierdo. Tinción de las arterias silvianas sólo en su porción proximal
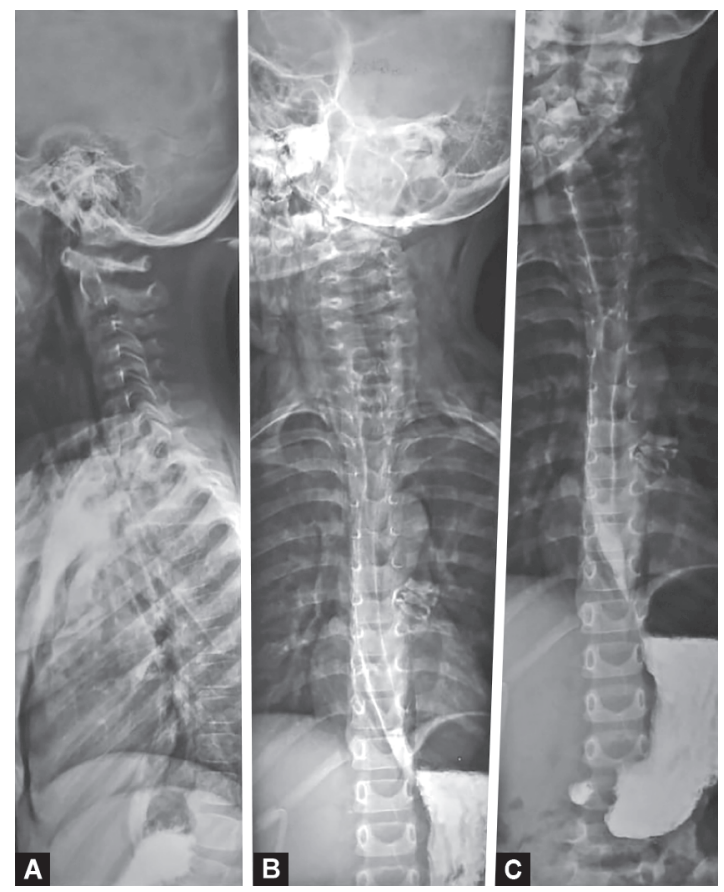

Figs 6 A to $C$ : Seriada esófago-gastro-duodenal

solicitan la derivación a un centro de mayor complejidad. La paciente ingresa al HNRG a las 5:00, trasladada por el sistema público de emergencias y luego de la evaluación por los servicios de Cirugía y Endoscopía se decide su internación en la unidad de cuidados intermedios. Se solicita radiografía de cuello y tórax (frente y perfil) Figure 5 y 6 , radiografía seriada esófago-gastro-duodenal y eco-doppler de vasos de cuello, que evidencian indemnidad de las estructuras vitales de la región cervical.

Se indica analgesia, profilaxis antibiótica con ceftriaxona y clindamicina y se mantiene conducta quirúrgica expectante. Por presentar buena evolución clínica, pasa a sala de internación general, externándose a los 4 días. 


\section{Discusión}

Los tres casos relatados presentaron evoluciones distintas, debido a los diferentes mecanismos de lesión y afectación de órganos, principalmente vasos arteriales y esófago.

Si bien el manejo inicial del paciente con TPC no difiere del de todo paciente traumatizado, en estas lesiones es fundamental establecer un diagnóstico y tratamiento oportunos y el punto crítico para su determinación es la evaluación del estado hemodinámico y el resultado de los estudios de imágenes: ante inestabilidad hemodinámica, compromiso de la vía aérea o sospecha de lesión laríngea, esofágica o vascular, se recomienda la cirugía de urgencia. ${ }^{10-12}$

Para establecer prioridades según la potencialidad de lesión de órganos vitales, el cuello se divide en tres zonas: ${ }^{1,4-7}$ zona I, desde la clavícula hasta el borde superior del cartílago cricoides; zona II, del cartílago cricoides al ángulo de la mandíbula, y zona III, desde el ángulo mandibular a la base del cráneo. Desde el punto de vista anatómico se divide en los sectores central, posterior y anterolateral. La importancia de esta división es que las heridas del triángulo posterior raramente involucran estructuras vitales, mientras que en el triángulo anterior esta probabilidad es muy superior. ${ }^{13}$

En el trauma de laringe, la tríada de signos clínicos es disfonía, enfisema subcutáneo y fractura palpable. La respuesta verbal positiva y apropiada del paciente indica una vía aérea permeable, con ventilación adecuada y suficiente perfusión cerebral. La intubación sólo está justificada en caso de obstrucción total de la vía aérea, dificultad respiratoria severa, fractura de laringe no diagnosticada y ruptura incompleta de la vía aérea superior. Se sugiere en primer lugar la intubación guiada por endoscopía flexible; si esta fracasa, la siguiente opción es la traqueotomía de urgencia y, si fallara, cricotiroidotomía quirúrgica. ${ }^{14,15}$ En cualquiera de los casos, es fundamental recordar la necesidad de inmovilización de la columna cervical. Si la lesión está por debajo de la membrana cricotiroidea deberá optarse por una traqueostomía de urgencia. ${ }^{16}$

Las lesiones de la arteria carótida se han informado en $0.7-6 \%$ de las heridas penetrantes de cuello y representan el $22 \%$ de las lesiones vasculares cervicales, asociándose a una mortalidad de 8.3-22\%. La arteria carótida común es la más frecuentemente lesionada, seguida por la carótida interna y externa. La mortalidad asociada a lesiones de la carótida común es más elevada que las de carótida interna, probablemente por la asociación con lesiones de la vena yugular interna (31\%). Los pacientes con estas lesiones presentan: shock (81\%), hemorragia activa (60\%), hematoma (33\%) y déficit neurológico (20\%). ${ }^{4,6,17,18}$ El manejo quirúrgico (reparación vs ligadura) es un tema controvertido. Algunas revisiones afirman que la morbimortalidad es significativamente más baja en los pacientes con reparación primaria (15\%) que en aquellos con ligadura arterial $(50 \%)^{4,6}$ y el resultado depende en buena medida del alto grado de sospecha. ${ }^{1}$

En los casos comentados encontramos tres presentaciones y evoluciones clínicas de severidad claramente diferente, que ordenaremos en forma creciente. En el caso 3, la lesión se sospechó por mecanismo y presentación clínica (enfisema subcutáneo, aerorragia cervical) pero no tuvo confirmación por imágenes ni exploración quirúrgica, razones por las cuales se optó por una estrategia conservadora. Uno de los aspectos más controvertidos en estos pacientes se relaciona con el manejo inicial de la vía aérea. De acuerdo con diferentes reportes, la proporción de pacientes con TPC que requiere intubación de urgencia oscila alrededor de
$10 \%$ aunque con alta variabilidad, dada la ausencia de guías de práctica claras ${ }^{19,20}$ y que este procedimiento no está exento de complicaciones, sobre todo en centros no especializados. En el caso presentado, por la estabilidad hemodinámica y la permeabilidad de la vía aérea se optó por no instrumentar la vía aérea con buen resultado.

En el caso 1, la paciente presentó una lesión extensa de la vía aérea pero sin lesiones arteriales asociadas, razón por la cual tuvo una evolución tórpida, principalmente asociada a la severidad de las lesiones, el impacto pulmonar de la enfermedad crítica (SDRA) y las complicaciones (NAV), pero con buenos resultados. La severidad de las lesiones puede estimarse con la escala AIS. De acuerdo con la versión de 1998, a la lesión traqueal le corresponde una severidad de grado 4, a la yugular un grado 3 y a la lesión tiroidea, un grado 2 , por lo cual sólo con las lesiones del cuello la paciente tuvo un puntaje NISS de 29.

En el caso 2, la asociación de la disrupción carotídea con ruptura de C5 y lesión de la arteria vertebral fue de tal severidad que el pronóstico estuvo marcado desde los primeros minutos. Si bien el paciente fue trasladado rápidamente y su tratamiento quirúrgico fue precoz, las posibilidades de una buena recuperación eran muy bajas. A la transección carotídea le corresponde una severidad por AIS de 5, a la lesión de la arteria vertebral un puntaje de 3 y a la del pedículo de $C 5,2$. El NISS resultante, sin tener en cuenta otras lesiones es 38. Este tipo de lesiones tiene muy alta letalidad ${ }^{21}$ y el caso presentado cumplía los criterios de Reva et al. ${ }^{22}$ para la ligadura carotídea.

\section{ConClusión}

EI TPC es poco frecuente en pediatría. La edad parece influir en el tipo de lesión, pero no afecta la mortalidad. La TC es el estudio diagnóstico imprescindible utilizado para el tratamiento selectivo. El tipo de lesión vascular y la hipotensión inicial se asocian independientemente con la mortalidad.

EI TPC requiere a menudo manejo quirúrgico de urgencia, ya que las lesiones de la vía aérea y de la circulación carotidea son lesiones que comprometen la vida. La anatomía de la región y la complejidad de su abordaje quirúrgico son los principales responsables de la dificultad en el manejo de estos pacientes. La mortalidad es alta durante la primera hora, por lo cual la mejora de los resultados depende de una adecuada articulación de todos los eslabones de la cadena de atención, desde la atención prehospitalaria hasta el manejo quirúrgico, pasando por un alto grado de sospecha de las lesiones.

\section{References}

1. Flores J, Ortiz PJ, Cervantes J. Trauma penetrante de cuello: ¿es confiable la exploración física para el diagnóstico de lesiones? An Med Asoc Med Hosp ABC 2000;45:6-12.

2. Abujamra $L$, Joseph $M M$. Penetrating neck injuries in children: a retrospective review. Pediatr Emerg Care 2003;19(5):308-313. DOI: 10.1097/01.pec.0000092575.40174.f3.

3. Stone ME, Farber BA, Olorunfemi O, et al. Penetrating neck trauma in children: an uncommon entity described using the National Trauma Data Bank. J Trauma Acute Care Surg 2016;80(4):604-609. DOI: 10.1097/TA.0000000000000976.

4. Magaña SI, Cabello PR, Melgoza MD, et al. Patrón de lesiones de cuello en el Hospital Central Militar. Cir Gen 2001;23:240-244.

5. Carreón Bringas R, González Bonilla R. Lesiones estructurales en trauma de cuello, reporte de dos años en un hospital de urgencias de la Ciudad de México. Trauma 2004;7:47-52. 
6. Allen CJ, Straker RJ, Tashiro J, et al. Pediatric vascular injury: experience of a level 1-trauma center. J Surg Res 2015;196(1):1-7. DOI: 10.1016/j.jss.2015.02.023.

7. Millán HM, Gómez BJ, Hernández HJ, et al. Manejo del trauma penetrante cervical de zona II. Hospital Clínico de Valdivia. Serie de casos clínicos. Cuad Cir (Valdivia) 2008;22(1):11-17. DOI: 10.4206/cuad. cir.2008.v22n1-02.

8. Fundación Trauma. Registro de Trauma. Informe 2018. Disponible en: https://publicaciones.fundaciontrauma.org.ar//Reportes/ ViewReporte?reporteld=64. Fecha de acceso 04/2019.

9. Illescas Fernández G. Manejo prehospitalario de urgencia del trauma de cuello. Trauma 2006;9:79-82.

10. Tessler RA, Nguyen $\mathrm{H}$, Newton $\mathrm{C}$, et al. Pediatric penetrating neck trauma: Hard signs of injury and selective neck exploration. J Trauma Acute Care Surg 2017;82(6):989-994. DOI: 10.1097/ TA.0000000000001407.

11. Rathlev NK, Medzon R, Bracken ME. Evaluation and management of neck trauma. Emerg Med Clin North Am 2007;25(3):679-694. DOl: 10.1016/j.emc.2007.06.006.

12. Burch JM, Franciose RJ, Moore EE. Trauma, En: Schwartz S, Shires T, Spencer F. Principios de Cirugía. Capítulo 6. $8^{\circ}$ ed., México. Interamericana 2006. 129187.

13. Barkana Y, Stein M, Scope A, et al. Prehospital stabilization of the cervical spine for penetrating injuries of the neck- is it necessary? Injury 2000;31(5):305-309. DOI: 10.1016/s0020-1383(99)00298-3.

14. Paniagua C. Manejo perioperatorio de lesiones de cuello. Rev Mex Anest 2008;31:183-186.
15. Shatney $\mathrm{CH}$, Brunner RD, Nguyen TQ. The safety of orotracheal intubation in patients with unstable cervical spine fracture or high spinal cord injury. Am J Surg 1995;170(6):676-680. DOI: 10.1016/ s0002-9610(99)80040-3.

16. Rajani RR, Ball CG, Montgomery SP, et al. Airway management for victims of penetrating trauma: analysis of 50,000 cases. Am J Surg 2009;198(6):863-867. DOI: 10.1016/j.amjsurg.2009.08.013.

17. Perea LL, Hazelton JP, Fox N, et al. Pediatric major vascular injuries: a 16-year institutional experience from a combined adult and pediatric trauma center. Pediatr Emerg Care 2018. DOI: 10.1097/ PEC.0000000000001642. Epub ahead of print. Available at: https:// pubmed.ncbi.nlm.nih.gov/30335690/.

18. Swendiman RA, Luks VL, Hatchimonji JS, et al. Mortality after adolescent firearm injury: effect of trauma center designation. J Adolesc Health 2020. DOI: 10.1016/j.jadohealth.2020.09.004S1054$139 \times(20) 30525-5$.

19. Youssef N, Raymer KE. Airway management of an open penetrating neck injury. CJEM 2015;17(1):89-93. DOI: 10.2310/8000.2013.131254.

20. Mandavia DP, Qualls S, Rokos I. Emergency airway management in penetrating neck injury. Ann Emerg Med 2000;35(3):221-225. DOI: 10.1016/s0196-0644(00)70071-0.

21. Lee TS, Ducic Y, Gordin E, et al. Management of carotid artery trauma. Craniomaxillofac Trauma Reconstr 2014;7(3):175-189. DOI: 10.1055/s0034-1372521.

22. Reva VA, Pronchenko AA, Samokhvalov IM. Operative management of penetrating carotid artery injuries. Eur J Vasc Endovasc Surg 2011;42(1):16-20. DOI: 10.1016/j.ejvs.2011.01.025. 